\title{
Current Drag in Capacitively Coupled Luttinger Constrictions
}

\author{
Yuli V. Nazarov \\ Department of Applied Physics and Delft Institute of Micro-electronics and Submicron-technology (DIMES), \\ Delft University of Technology, 2628 CJ Delft, The Netherlands \\ D. V. Averin \\ Department of Physics, State University of New York, Stony Brook, New York 11794
}

(Received 15 May 1997; revised manuscript received 1 December 1997)

\begin{abstract}
We study the current drag in the system of two electrostatically coupled finite 1D electron channels. We present the perturbation theory results along with the results for two nonperturbative regimes. It is shown that the drag can become absolute; that is, the currents in the channels are equal in a finite window of the bias voltages. [S0031-9007(98)06648-4]
\end{abstract}

PACS numbers: 71.10.Pm, 73.23.Ad

The recent experiments with two capacitively coupled two-dimensional electron gases (2DEG) [1] demonstrated that, owing to Coulomb interaction, electrons moving in one of the 2DEG drag the electrons of the adjacent 2DEG. Therefore the system works as a dc current transformer. The transformation coefficient is, however, much smaller than one [2,3]. In specially designed coupled 1D arrays of ultrasmall tunnel junctions Coulomb interaction can lead to the absolute current drag [4]. This means that the electric currents in two capacitively coupled circuits are equal in magnitude in a certain region of voltages applied to the circuits, and the system may work as a current copier. This prediction has been recently confirmed [5] experimentally.

The mechanism of the absolute current drag in small tunnel junctions is, however, quite different from the momentum-transfer mechanism in the 2DEG. The aim of the present work is to show that in the 1D electron channels, the momentum-transfer mechanism can also lead to the absolute current drag. We consider a system of two channels of finite length $L$ that are adiabatically connected to the reservoirs of effectively noninteracting electrons (Fig. 1). The channels are assumed to be coupled only by the Coulomb interaction. We assume no impurities in the channels that would cause electron backscattering. Recent advances [6] demonstrate that such systems can be successfully fabricated in the near future.

We stress that in the system of two wires studied in this work the drag couples dissipative currents, and the presence of the reservoirs is of crucial importance. Another phenomenon, drag of persistent currents, should occur in closed 1D loops penetrated by magnetic flux [7].

To get an intuitive feeling of how the absolute current drag can occur in such a system, let us consider the case of strong electron repulsion. In this case, electrons in each channel form a rigid Wigner lattice. Provided the channels are close to each other, the repulsion coordinates not only the positions of electrons inside the same channel but also the positions of neighboring electrons in the other channel (Fig. 1). Now, if the electrons in one of the channels move, electrons in the other channel must follow their motion, and the electric currents in the channels are equal.

Such a simple model says very little about realistic 1D channels where electrons are subjected to strong quantum fluctuations. Below we develop a consistent theory of current drag which accounts for these fluctuations. The main prediction of the theory is that the (almost) absolute current drag survives quantum fluctuations. It can occur even if the repulsive interaction is weak provided the channels are sufficiently long.

We model each of the two coupled 1D conductors as "Luttinger constriction" with spinless electrons, i.e., use the standard Hamiltonian [8]

$$
H=\int \frac{d x}{2 \pi} u\left[g \Pi^{2}+\frac{(\nabla \Theta)^{2}}{g}\right]
$$

Here $\Theta(x)$ is the displacement of electrons in the conductor normalized in such a way that the local fluctuation of

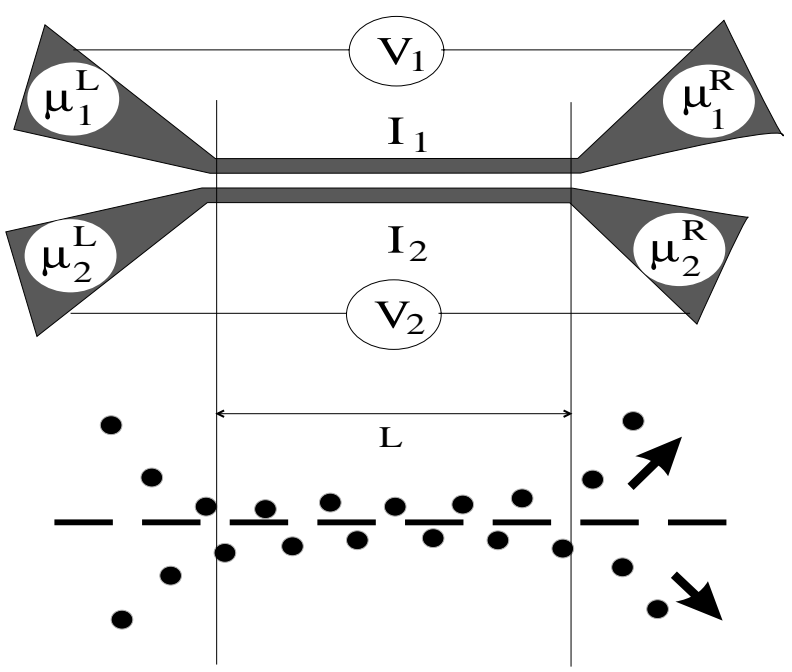

FIG. 1. The system under consideration. Two 1D channels are open to reservoirs and separated by insulating barrier. The electrostatic repulsion coordinates the positions of electrons (black circles) in the channels, the fact that can lead to the absolute current drag. 
electron density is $\delta n=-\nabla \Theta / \pi$, and $\Pi(x)$ is the conjugated momentum density: $\left[\Theta(x), \Pi\left(x^{\prime}\right)\right]=i \pi \hbar \delta(x-$ $\left.x^{\prime}\right)$. The velocity $u$ and interaction strength $g=u_{F} / u$ are piecewise constants of $x$ : in the reservoirs they assume their noninteracting values $u_{F}$ and 1 .

The relevant part of the interaction of the two conductors responsible for the momentum transfer between them can be written as

$$
\begin{aligned}
H_{c}=J \int_{-L / 2}^{L / 2} \frac{d x}{2 \pi} \cos \left\{2\left(k_{F 1}-k_{F 2}\right) x\right. \\
\left.+2\left[\Theta_{1}(x)-\Theta_{2}(x)\right]\right\},
\end{aligned}
$$

where subscripts 1 and 2 refer to the two conductors, $k_{F j}=\pi n_{j}$ are the Fermi wave vector, and $J$ is the interaction constant depending on the specific electrostatic configuration of the system.

The model we just formulated is mathematically similar to that of Luther and Emery [9]. The electrons of two spin directions in their model correspond to electrons in two different wires in our model. The essential difference between the two models is that we deal with wires that interact within a finite length $L$. Below we describe three distinct drag regimes: (i) perturbative regime (weak drag), (ii) nonperturbative weak coupling regime (strong drag), and (iii) strong coupling regime (absolute drag), the latter corresponding to perfect latching.

When the coupling is weak, electrons in the two channels move independently and can carry generically different currents $I_{1}$ and $I_{2}$. When $I_{1} \neq I_{2}$, momentum transfer between the channels leads to the appearance of an extra voltage drop $\delta V_{j}$ across each channel, in addition to the "ideal" Landauer value $h I_{j} / e^{2}$. Those can be found from the difference of chemical potentials at the ends of the channels,

$$
\delta V_{j}=\frac{\kappa_{j}}{e}\left\langle\delta n_{j}(-L / 2)-\delta n_{j}(L / 2)\right\rangle,
$$

$\kappa$ 's being compressibilities of electron liquids in the channels. Writing the equations of motion for phases $\Theta$ that follow from the Hamiltonian $H=H_{1}+H_{2}+H_{c}$ :

$$
\begin{aligned}
\ddot{\Theta}_{j}-\left[u_{j}^{2} \Theta_{j}^{\prime}\right]^{\prime}=(-1)^{j} J u_{F} \sin \{ & 2\left(k_{F 1}-k_{F 2}\right) x \\
+ & \left.2\left[\Theta_{1}(x)-\Theta_{2}(x)\right]\right\},
\end{aligned}
$$

and using the fact that in the stationary case $\langle\ddot{\Theta}\rangle=0$, we get that $\delta V_{2}=-\delta V_{1} \equiv V$, and

$$
\begin{aligned}
V & =\int_{-L / 2}^{L / 2} d x \frac{F(x)}{e}, \\
F(x) & =J\left\langle\sin \left\{2\left(k_{F 1}-k_{F 2}\right) x+2\left[\Theta_{1}(x)-\Theta_{2}(x)\right]\right\}\right\rangle .
\end{aligned}
$$

In the first order of the perturbation theory in $H_{c}$ we get

$$
\begin{aligned}
F(x)= & \frac{J^{2}}{2} \operatorname{Re} \int_{-L / 2}^{L / 2} d x^{\prime} \\
& \times \int_{0}^{\infty} d t e^{i\left[k\left(x-x^{\prime}\right)+\Omega t\right]} e^{A}\left(e^{B}-e^{-B}\right),
\end{aligned}
$$

where $A\left(x, x^{\prime}, t\right)=\left\langle\left[\varphi, \varphi^{\prime}\right]_{+}-\varphi^{2}-\varphi^{\prime 2}\right\rangle / 2$ and $B(x$, $\left.x^{\prime}, t\right)=\left\langle\left[\varphi, \varphi^{\prime}\right]\right\rangle / 2$. Here $\varphi=\varphi(x, t), \varphi^{\prime}=\varphi\left(x^{\prime}, 0\right)$, and the phase $\varphi(x, t)$ is introduced by the relation:

$$
\begin{aligned}
2\left[\Theta_{1}(x, t)-\Theta_{2}(x, t)\right] & =\Omega t+\varphi(x, t), \\
\Omega & =2 \pi\left(I_{1}-I_{2}\right) / e .
\end{aligned}
$$

The Fermi-momentum difference $k=2\left(k_{F 1}-k_{F 2}\right)$ is assumed to be small $k \ll k_{F 1,2}$. The average $\langle\ldots\rangle$ in the definitions of the correlators $A$ and $B$ is taken over the equilibrium fluctuations of $\varphi$.

The equilibrium correlators $A, B$ can be expressed in a standard way in terms of the spectral densities $\rho_{j} \equiv$ $(1 / \pi) \operatorname{Im} G_{R}^{(j)}\left(x, x^{\prime}, \omega\right)$,

$$
A\left(x, x^{\prime}, t\right) \pm B\left(x, x^{\prime}, t\right)=2 \sum_{j=1,2} \int d \omega\left[e^{i \omega t} \rho_{j}\left(x, x^{\prime}, \omega\right)\left(\operatorname{coth} \frac{\omega}{2 T} \pm 1\right)-\rho_{j}(x, x, \omega)-\rho_{j}\left(x^{\prime}, x^{\prime}, \omega\right)\right] .
$$

The retarded Green's functions $G_{R}^{(j)}$ of the phases $\Theta_{j}$ can be solved explicitly for our model of piecewise constant $u(x)$ and give

$$
\rho\left(x, x^{\prime}, \omega\right)=\frac{g^{2}}{\omega} \frac{\left(1+g^{2}\right) \cos \nu\left(x-x^{\prime}\right)+\left(1-g^{2}\right) \cos \nu L \cos \nu\left(x+x^{\prime}\right)}{\left(1+g^{2}\right)^{2} \sin ^{2} \nu L+4 g^{2} \cos ^{2} \nu L}, \quad \nu=\omega / u
$$

Equation (5) combined with Eqs. (8) and (9) allows us to find the induced voltage $V$ as a function of the current difference $I=I_{1}-I_{2}$ in various regimes. We consider first identical channels at zero temperature. In the noninteracting case $g=1$ we get then from Eq. (5)

$V=\frac{\pi J^{2} L u \Omega^{2}}{2 e E_{F}^{4}} \int_{0}^{L \Omega / u} \frac{d x}{x} \frac{\sin x-x \cos x}{x^{2}}\left(1-\frac{x u}{L \Omega}\right)$, where $E_{F}$ is the cutoff frequency of the order of Fermi energy. For $\Omega \ll u / L$, the voltage $V$ grows as $\Omega^{3}$, this growth slowing down to $\Omega^{2}$ at larger $\Omega$.

At small frequencies the spectral density (9) is dominated by the leads and approaches its noninteracting value $1 / 2 \omega$. This means that the dependence $V \propto \Omega^{3}$ is valid at $\Omega \ll g u / L$ for any $g$.

To see how $V$ behaves at large current differences $(\Omega \gg u / L)$ we can average the rapid oscillations of 


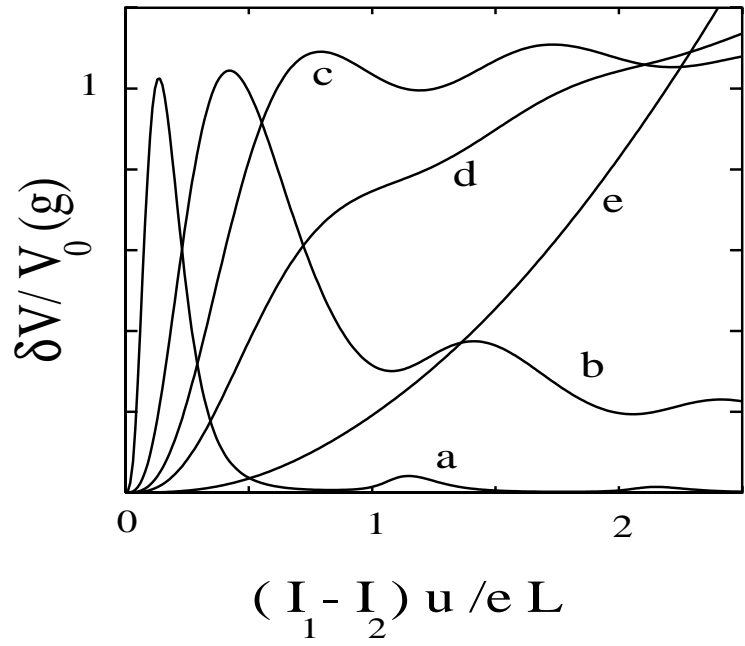

FIG. 2. Induced voltage difference $\delta V$ in the perturbative regime for constrictions with $k_{F 1}=k_{F 2}$, normalized to $V_{0}(g) \equiv J^{2} u L E_{F}^{-2}\left(u / E_{F} L\right)^{4 g-2}$. The curves $a, b, c, d, e$ correspond to $g=0.1,0.3,0.5,0.6,1.0$, respectively.

spectral density (9) with the period $u / L$. We get for $V$

$$
\begin{aligned}
V & =F L \\
& =\frac{J^{2} L u \pi^{2}}{\Gamma(g)^{2} E_{F}^{2}}\left(\frac{\sqrt{\Omega^{2}-u^{2} k^{2}}}{2 E_{F}}\right)^{4 g-2} \Theta\left(\Omega^{2}-u^{2} k^{2}\right),
\end{aligned}
$$

$g=\left(g_{1}+g_{2}\right) / 2$. We see that the interaction inside the constriction changes drastically the behavior of $V$ as a function of $\Omega$ giving rise to powerlike dependence. The voltage $V$ decreases at large $I$ for $g<1 / 2$, so that there is optimum $I \simeq e u / L$ for which the induced voltage is maximum. If we take oscillating terms into account, the decrease at large current differences is nonmonotonous, the voltage oscillates with $\Omega$ with the period $u / L$. This resembles density of states behavior in a finite Luttinger constriction [10]. All of these features can be seen in Fig. 2 which presents the results of numerical calculations of $V$ as a function of $I_{1}-I_{2}$ from Eqs. (6)-(8).

Another important feature of Eq. (11) is that the voltage $V$ grows with increasing $L$ at any given current difference $\Omega$ regardless of $g$. This suggests that Eq. (11) is valid only for sufficiently short channels. In longer channels the distribution of the voltage along the channel and the difference of electron densities associated with it in the two channels should be determined self-consistently. The variation of the density difference can be described by the steady-state form of Eq. (4) which can be written as follows:

$$
k^{\prime}(x)=\frac{4 g}{u} F(k(x), \Omega) .
$$

Here the force $F$ is given by Eq. (11) but now with local $k(x)$. In simple terms, Eq. (12) describes how the friction between the moving liquids compresses one of them and stretches another one, and how this compression/stretch affects the magnitude of the friction itself.

Equation (12) with the force given by Eq. (11) shows that in long channels, $L \gg L_{\Omega}$, where $L_{\Omega}^{-1} \equiv 16 g J^{2} u\left(\Omega / 2 E_{F}\right)^{4 g} / \Omega^{3}$ is the characteristic length of variation of $k(x)$, the density difference approaches the value $\Omega / \pi u$ and saturates. This means that the extra voltage difference drops at distances of the order of $L_{\Omega}$ near the edge of the interacting region and is equal to $h\left(I_{1}-I_{2}\right) / e^{2} 2 g$. The total voltage drops across the channels are given then by "quasi Landauer" relations

$$
\begin{aligned}
& \frac{e^{2} V_{1}}{h}=I_{1}+\frac{1}{2 g}\left(I_{1}-I_{2}\right), \\
& \frac{e^{2} V_{2}}{h}=I_{2}+\frac{1}{2 g}\left(I_{2}-I_{1}\right) .
\end{aligned}
$$

Another nonperturbative regime in which the currents in the two channels are almost precisely equal is the limit of strong coupling of the two short channels with $L \ll L_{Q}$. This regime corresponds to the classical limit of the sine-Gordon model [11]. In the limit of $g \rightarrow 0$, one can treat $\varphi$ as (almost) a classical variable that takes on $2 \pi \times$ integer values. In our original model, this means that the Luttinger liquids, or electron chains, are strictly correlated. However, they can move together and carry equal currents in both constrictions. Since $\delta V_{1}=-\delta V_{2}$ from Eq. (3), we obtain $I_{1}=I_{2}=e^{2}\left(V_{1}+V_{2}\right) / 2 h$.

This is, of course, an ideal situation, and at finite $g$ and $L$ the currents are not precisely equal. Let us show that, in the wide region of parameters, the current difference is exponentially small. To this end, we switch from the Hamiltonian to Lagrangian in imaginary time;

$$
S[\varphi(x, \tau)]=\int \frac{d x d \tau}{2 \pi}\left[\frac{1}{8 g}\left(u \varphi^{\prime 2}+\frac{\dot{\varphi}^{2}}{u}\right)+J \cos \varphi\right] .
$$

Equation (7) shows that the shift of $\varphi$ at the boundary by $4 \pi$ corresponds to transfer of one electron into the channel 1 accompanied by the transfer of an electron out of the channel 2. This suggests that in order to describe the interaction of the electron channels with the electrodes, we should add to the Lagrangian the boundary term

$$
\begin{aligned}
S_{b}=\int \frac{d \tau}{4 \pi}\left[\left(\mu_{2}^{R}-\mu_{1}^{R}\right) \varphi(-L / 2)\right. \\
\left.+\left(\mu_{1}^{L}-\mu_{2}^{L}\right) \varphi(L / 2)\right],
\end{aligned}
$$

where $\mu_{1,2}^{L, R}$ are the chemical potentials of the left-right electrode of the channels 1 and 2.

What are the processes that spoil the ideal current copying? Obviously, those are slips of $\varphi$ by $2 \pi$. If the voltages applied to the constriction are sufficiently low, the slip should happen at once throughout the interacting region. Otherwise a soliton would have been created within the interacting region as a result of the 


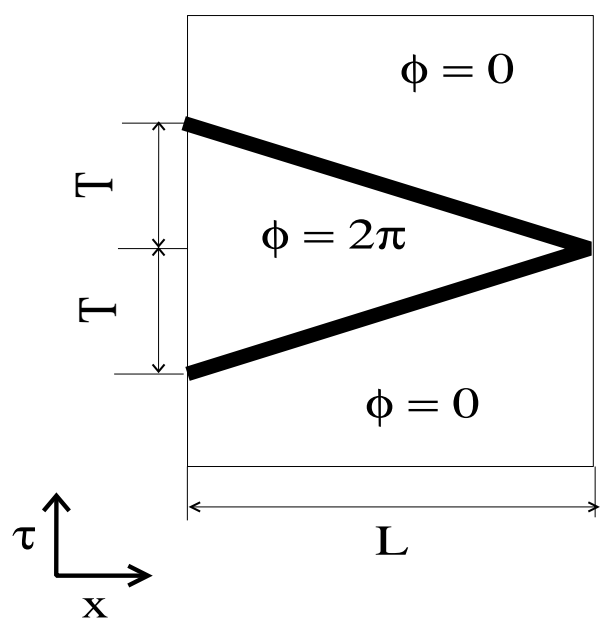

FIG. 3. Soliton tunneling via the interacting region. The optimal configuration of $\varphi(x, \tau)$ corresponds to three regions of almost constant $\varphi$ separated by V-shaped soliton "wall" (thick line).

slip. This would cost energy $E_{s}=\sqrt{J u / \pi g}$ and is forbidden by the energy considerations. The sudden slip throughout the interacting region may be viewed as a virtual soliton transfer via the "barrier" $E_{S}$, provided the barrier length exceeds the soliton size $\sqrt{u / J g}$. The rate of such a process is determined by the saddle-point configuration of $\varphi(x, \tau)$ as sketched in Fig. 3. The action has contributions from the two soliton "walls" and the boundary term, $S=2 E_{S} \sqrt{T^{2}+(L / u)^{2}}-2 T \mu$, where $\mu=\max \left(\left(\mu_{1}^{L}-\mu_{2}^{L}\right) / 2,\left(\mu_{1}^{R}-\mu_{2}^{R}\right) / 2\right)$. Minimization of $S$ with respect to $T$ yields

$$
S_{0}=2 \sqrt{E_{s}^{2}-\mu^{2}} L / u,
$$

and $I_{1}-I_{2} \propto \exp \left(-S_{0}\right)$. We see that the current difference is suppressed exponentially provided $L \gg u / E_{s}$ and $\mu_{L, R}<E_{s}$. The common wisdom of the sine-Gordon model [12] suggests that these results are, in fact, valid not only in the classical limit $g \rightarrow 0$ but also at any $g<1$, provided $E_{s}$ is properly renormalized corresponding to the actual soliton energy $E_{s}(g) \propto E_{S}\left(E_{S} / E_{F}\right)^{g / 1-g}$ with the coefficient depending on details of the cutoff.

In conclusion, we have considered current drag in the system of two capacitively coupled Luttinger constrictions. We have presented perturbative results and have found two nonperturbative regimes. In one of the regimes, the system works as an ideal current copier of exponential accuracy. Both nonperturbative regimes can be realized in constrictions made of the heterostruc- tures with coupled 2DEG [1,3]. Indeed, the crude estimate of interaction parameter $g$ for Coulomb electron-electron interaction screened at distance $D$ [13], $g^{-2}=1+2 \ln (8 D n) / \pi^{2} n a_{B}$, shows that for reasonable values of $D \simeq 100 \mathrm{~nm}, g=1 / 2$ corresponds to electron concentration $n \simeq(50 \mathrm{~nm})^{-1}$. In this case, for constrictions separated by $d \simeq 10 \mathrm{~nm}$ (as in the 2D experiment [3]) the maximum exponent (16) is $S \simeq 2 L / \pi\left(d a_{B}\right)^{1} / 2$ and the drag should become absolute already in relatively short constrictions with $L \simeq 0.3 \mu \mathrm{m}$.

We thank A.A. Odintsov for his contribution at preliminary stages of this work. We are indebted to $\mathrm{S}$. Tarucha for his communications that gave an impetus to the present research, and to G. E. W. Bauer and M.P. A. Fisher for very instructive discussions of the results. This work has been made possible by financial support of North Atlantic Treaty Organization, Grant No. 950279.

[1] P. M. Solomon, P. J. Price, D. J. Frank, and D.C. La Tulipe, Phys. Rev. Lett. 63, 2508 (1989); T. J. Gramila, J.P. Eisenstein, A. H. MacDonald, L.N. Pfeiffer, and K. W. West, Phys. Rev. Lett. 66, 1216 (1991).

[2] A. Kamenev and Yu. Oreg, Phys. Rev. B 52, 7516 (1995); K. Flensberg et al., Phys. Rev. B 52, 14761 (1995).

[3] M.P. Lilly, J.P. Eisenstein, L. N. Pfeiffer, and K.W. West, Phys. Rev. Lett. 80, 1714 (1998).

[4] D. V. Averin, A. N. Korotkov, and Yu. V. Nazarov, Phys. Rev. Lett. 66, 2818 (1991).

[5] M. Matters, J. J. Versluys, and J.E. Mooij, Phys. Rev. Lett. 78, 2469 (1997); P. Delsing, D. B. Haviland, and P. Davidsson, Czech. J. Phys. 46, 2359 (1996).

[6] S. Tarucha, T. Honda, and T. Saku, Solid State Commun. 94, 413 (1995); A. Yacoby, H. L. Stormer, Ned S. Wingreen, L. N. Pfeiffer, K. W. Baldwin, and K. W. West, Phys. Rev. Lett. 77, 4612 (1996).

[7] A. G. Rojo and G. D. Mahan, Phys. Rev. Lett. 68, 2074 (1992); T. V. Shahbazyan and S.E. Ulloa, Phys. Rev. B 55, 13702 (1997).

[8] F. D. M. Haldane, Phys. Rev. Lett. 47, 1840 (1981); for review see, e.g., J. Voit, Rep. Prog. Phys. 58, 997 (1995).

[9] A. Luther and V. J. Emery, Phys. Rev. Lett. 33, 589 (1974).

[10] Yu. V. Nazarov, A. A. Odintsov, and D. V. Averin, Europhys. Lett. 37, 213 (1997).

[11] S. Coleman, Phys. Rev. D 11, 2088 (1975), and references therein.

[12] R. F. Dashen, B. Hasslacher, and A. Neveu, Phys. Rev. D 11, 3424 (1975); A. Luther, Phys. Rev. B 14, 2153 (1976).

[13] L. I. Glazman, I. M. Ruzin, and B.I. Shklovskii, Phys. Rev. B 45, 8454 (1992). 